\title{
Early fault prediction and detection of hydrocephalus shunting system
}

\author{
Abdel Rahman Alkharabsheh ${ }^{1}$, Lina Momani ${ }^{2}$, Waleed Al-Nuaimy ${ }^{3}$, Jafar Ababneh ${ }^{1}$, \\ Tariq Alwada'n', Abeer Hawatmeh ${ }^{2}$ \\ ${ }^{1}$ Faculty of Science and Information Technology, The World Islamic Sciences and Education Technology, Amman, Jordan \\ ${ }^{2}$ Mechatronics Engineering Department, Faculty of Engineering and Technology, Al-Balqa' Applied University, Amman, Jordan \\ ${ }^{3}$ Department of Electrical Engineering, University of Liverpool, Liverpool, UK \\ Email:abdkr@hotmail.com, 1momani@hotmail.com
}

Received 15 October 2012; revised 13 January 2013; accepted 27 January 2013

\begin{abstract}
Trends of various intracranial pressure (ICP) parameters for high pressure hydrocephalus patients are utilized to detect various shunt faults in their early stages, as well as, to monitor the effect of such faults on shunt performance. A method was proposed to predict the time required for ICP to be abnormal and for the valve to reach full blockage condition. Furthermore, an auto valve schedule updating method is proposed and used to temporarily deal with detected faults until the patient is checked up by his/her physician. The proposed algorithms were evaluated using numerical simulation.
\end{abstract}

Keywords: Hydrocephalus Shunts; Shunt Malfunctions; Faults Detection

\section{INTRODUCTION}

Hydrocephalus is a neurological disorder whereby the cerebro-spinal fluid (CSF) surrounding the brain builds up, causing severe pain and swelling of the head [1]. This is particularly prevalent in infants, and is becoming more common.

Shunts were used for decades to treat hydrocephalus patients, where mechanical valves were the popular type for draining the CSF. The problem is these valves have serious drawbacks e.g. overflow, low long-term accuracy, drift, low durability.

In addition, almost the same treatment used for all hydrocephalus patients without taking into consideration patient's personal factors (such as medical history, sleep patterns and lifestyle) and it cannot handle real-time patient satisfaction and emergency situations. This makes the conventional mechanical shunt satisfying less of $50 \%$ of its patients. Nowadays, and after long history with such valves, it is found that, it is the proper time to use electronic valves.

\subsection{Shunt Drawbacks}

This passive operation causes many problems such as overdraining and underdraining. The management of shuntrelated problems and disorders has become a defacto subspecialty within neurosurgery. Although it is clear that the treatment of hydrocephalus was vastly improved with the introduction of the differential pressure valve, probably one of the most refractory problems of CSF shunt diversion has been that of overdrainage. Overdraining occurs when the shunt allows CSF to drain from the ventricles more quickly than it is produced. This overdraining can cause the ventricles to collapse. Underdraining occurs when CSF is not removed quickly enough and the symptoms of hydrocephalus recur. These problems may have dramatic effects on the patients such as brain damage.

Despite recent advances in valve technology, these shunts do not suit many hydrocephalus patients. This can be realised from the considerable high shunt revision and failure rates: between $30 \%$ and $40 \%$ of all CSF shunts placed in paediatric patients fail within the first year [2-5] and it is not uncommon for patients to have multiple shunt revisions within their lifetime.

Shunt systems are not perfect devices and complications often arise. Complications may include mechanical failure, infections, obstructions, and the need to lengthen or replace the catheter. Generally, shunt systems require monitoring and regular medical follow up. The diagnosis of such malfunctions can be both difficult and perplexing even for the experienced clinician.

\subsection{The Need for Mechatronic Shunt}

Symptoms of various shunt complications (seizures, a significant change in intellect, school performance, or personality) can be very similar and difficult to spot thus complicating the diagnosing process. On the other hand, the possible presentations of acute shunt malfunction in 
early stages are innumerable for many reasons such as the lack of non invasive intracranial pressure (ICP) and flow monitoring. In addition, shunt malfunctions might be present even if they have not shown on a CT or MRI scans and also the number of these should be minimized due to the use of radiation and are therefore not desirable for regular use. The early detection and recognition of shunt malfunctions, to prevent or minimize complications and maximize shunt functioning, has long been accepted as a desirable goal in the treatment of hydrocephalus. Despite ICP monitoring currently being an invasive procedure, patients with hydrocephalus may need repeated episodes of monitoring months or years apart. This involves hospitalization, patient suffering, and might endanger patient's life.

The patient needs to visit a hospital or meet a consultant for diagnosing every time he/she complains from symptoms. And since these symptoms (e.g. headache, vomiting, fever) are similar to the symptoms of other medical problems, the hydrocephalus patient would worry every time he/she has such symptoms. In this case, the patient will only be assured if he/she contacted a physician. This involves waste of patient and physician time and cost if the cause of the symptoms was not due to shunt malfunction.

Biomedical implants require a clean and medically safe source of energy to perform their operations. Limited battery life causes the impracticality, health risks and expense of operating on patients for the mere purpose of replacing the battery.

Up to this moment, the implanted battery needs to be replaced when it loses its capacity. Thus minimising power consumption is essential for any implantable device. In addition, memory size limitation is another obstacle that faces biomedical implants [6].

To address the above problems, an intelligent wireless shunting system needs to be designed. By having such system, the hospitalisation periods and patient suffering and inconvenience are reduced, the quality of treatment is improved and better understanding of intracranial hydrodynamics is established thanks to the valuable resource of ICP data collected by an implanted sensor.

\section{MECHATRONIC SHUNTING SYSTEM}

\subsection{Description}

An intelligent implantable wireless hydrocephalus shunting system was proposed by Momani et al. [7]. It aims to replace the passive shunt with an intelligent microcontroller that wirelessly communicates with a hand-held device operated by the patient, or on the patient's behalf by a clinician or guardian. This device would have a graphical user interface, microcontroller and an RF interface to communicate with the user and the implantable wireless electronic shunt. The implantable wireless shunting system would consist of electronic valve, intracranial pressure sensor, flowmeter, microcontroller and RF transceiver. An embedded implantable shunting software would autonomously regulate the electronic valve. The intelligent system is demonstrated in Figure 1.

The mechatronic shunting system is now under investigation [7]. One of the main benefits of using such system is the ability to non invasively monitor hydrocephalus patients in real time. Based on the proposed design of such shunting system, a daily ICP report would be generated by the implanted system and wirelessly send to the patient device. This report would include useful information that is used to derive ICP and flow parameters such as mean ICP, mean valve flow, mean absolute deviation.

\subsection{Controlling Methodology of Mechatronic Valve}

The principle of controlling mechatronic valve is by using a time based schedule. Such schedule would incur many disadvantages, e.g. overdrainage/underdrainage, if its selection is arbitrary. In order to optimize the usefulness of such a valve, a method and approach to manage and control such valve is essential. To eliminate previous disadvantages and make such a valve fully functional, a method is needed to reprogram, modify or replace the time based schedule of this valve dynamically.

An intelligent shunting system can be used to autonomously regulate the mechatronic valve according to certain valve schedule and update it based on the intracranial pressure when such data is available. In such a system, ICP readings and other sensory inputs such as patient feedback, would help in tuning the treatment and enabling the intervention of the medical practitioner to update and manually adapt the schedule. In addition, this system can be used to self diagnose its implanted components based on ICP data and valve flow measurements. Such diagnosis would improve the treatment and reduce patient suffering.

Early faults detection in biomedical systems is of vital importance in reducing the risk on patient's life. Its importance is increased in case of implanted system. Leading to increase system reliability, avoid hospitalisation, and reduce patient suffering.

The methods used to date for diagnosing shunt malfunctions have been based on clinical presentation of such malfunctions, clinical data, imaging techniques and evaluation of valve function in mechanical terms. Unfortunately, symptoms of various shunt complications can be very similar and difficult to spot thus complicating the diagnosing process. 


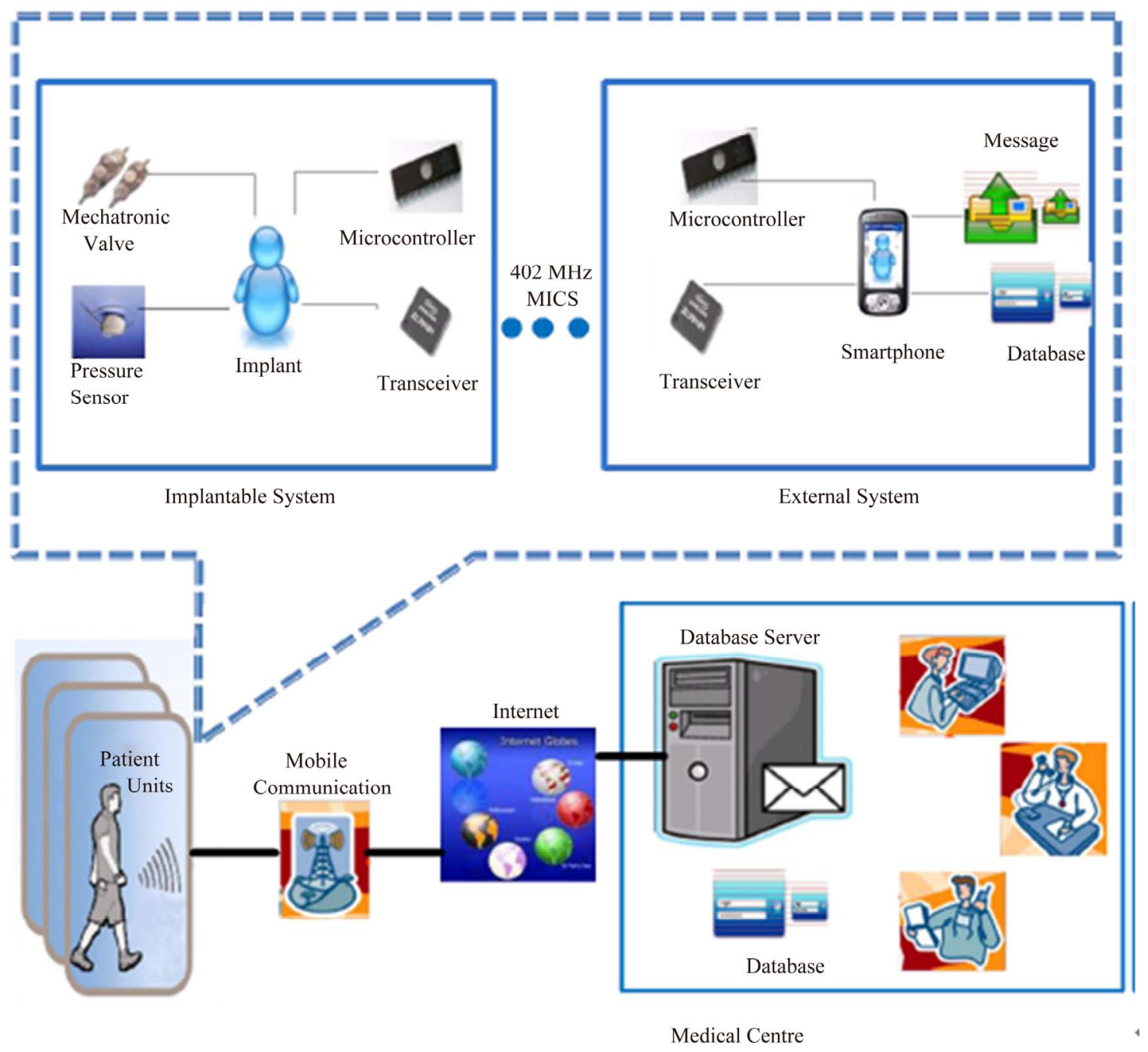

Figure 1. The intelligent shunting system.

\subsection{Common Faults of Shunting Systems}

Shunt systems are not perfect devices and complications often arise. Complications may include mechanical failure, infections, obstructions, and the need to lengthen or replace the catheter. Generally, shunt systems require monitoring and regular medical follow up. Shunt malfunction could be due to one of the following reasons: it's blocked with by products of the CSF, some of its components get disconnected, it breaks (usually the distal end), any of the catheters come out of their place, the shunt drains less fluid than it is supposed to or the shunt drains more fluid than it is supposed to.

The symptoms of various shunt complications can be very similar thus complicating the diagnosing process. A shunt is said to be malfunctioning if it is draining at an inappropriate rate. Underdrainage means that insufficient CSF is removed from the ventricles, so that symptoms are not fully alleviated. Also damaging can be overdrainage, which may result in collapse of the ventricles.
If this is very rapid, the brain may be torn away from the inner surface of the skull, causing bleeding, which may induce compression of the brain. Less severe overdrainage can result in low ICP with symptoms including severe headaches. Incorrect drainage is often the result of blockage/leakage, either of the catheter or of the tubing. Blockage of the catheter is more common and is a result of a buildup of cells (including those thought to produce CSF) in the holes of the catheter.

Disconnection of the shunt is considered the second most common cause of shunt failure after valve blockage. Disconnection may occur at any site of connection along the course of the tubing. This is usually related to improper technique (loose ligature) or excessive strain along the shunt tube between two points of fixation.

\subsection{Diagnosis of Shunting Faults}

Shunt malfunction is one of the most common clinical problems in paediatric neurosurgery. The diagnosis of 
such malfunctions can be both difficult and perplexing even for the experienced clinician. The methods used to date have been based on clinical presentation of shunt malfunctions, clinical data, imaging techniques and evaluation of valve function in mechanical terms. Unfortunately, symptoms of various shunt complications (seizures, a significant change in intellect, school performance, or personality) can be very similar and difficult to spot thus complicating the diagnosing process. On other hand, the possible presentations of acute shunt malfunction in early stages are innumerable for many reasons such as the lack of non invasive intracranial pressure (ICP) and flow monitoring. In addition, shunt malfunctions might be present even if they have not shown on a CT or MRI scans and also the number of these should be minimized due to the use of radiation and are therefore not desirable for regular use. The early detection and recognition of shunt malfunctions to prevent or minimize complications and maximize shunt functioning has long been accepted as a desirable goal in the treatment of hydrocephalus.

One method of achieving this goal is through the use of real time noninvasive ICP monitoring for hydrocephalus patients. The purpose of this study is to develop an effective method to give endowment to the current implanted shunting system with ICP sensor, flowmeter and transceiver to be able to make a real time self-diagnosis for the shunt. In this method, some features are extracted from intracranial pressure signals which are collected via an implanted ICP sensor and valve flow measurements which are measured using implanted flowmeter.

Thus, ICP monitoring allows the judicious use of interventions such as ICP signal information, implanted shunt diagnosis information and hydrocephalus management information. This may avoid potentially harmful and overly aggressive treatment such as regular shunt revision and invasive ICP monitoring method. Long-term monitoring of intracranial pressure (ICP) is limited by the lack of an implantable sensor with low drift as well as the power needed for the implanted components. In this case, short period of time has been used to collect small samples of ICP readings. One of the main objectives of this work is to optimize such sample to be more efficient in management and treatment of hydrocephalus, as well as diagnosis of shunting system.

One of the main challenges, for hydrocephalus researchers as well as for physician or consultant to manage the hydrocephalus and to diagnose the shunt malfunction, is lack of ICP data. To deal with such problem and to build a useful hydrocephalus database, the proposed mechatronic shunting system would monitor ICP and store data in the patient device as a patient record. Such record would be used to constitute a hydrocephalus database. This database can play a vital role in treatment of hydrocephalus and shunt diagnosis process.

\subsection{Contribution}

The first contribution of developing such method is enabling the system to early detect and warn of any expected shunt fault before it is occurred based on the parameters' trends and patterns. The trends of the parameters are utilized to monitor the behaviour of ICP signals and then to conclude the current status of the shunt components. In addition, it is used to predict the possibility of any future fault in shunt components. A block diagram of the proposed system is shown in Figure 2.

Trend detection algorithm is proposed and applied in this study to detect the trends of the derived parameters. In addition, simple predictive procedures are developed to estimate the required period of time needed for (ICP) to be in abnormal range.

The strength of relation between trends of the parameters and the degree of valve blockage is used to predict any future fault in the valve and calculate the expected time that is required to make a valve in full blockage status. The proposed method is also used to deal with various shunt complications such as valve leakage problem.

Since the mechatronic shunting system would enable

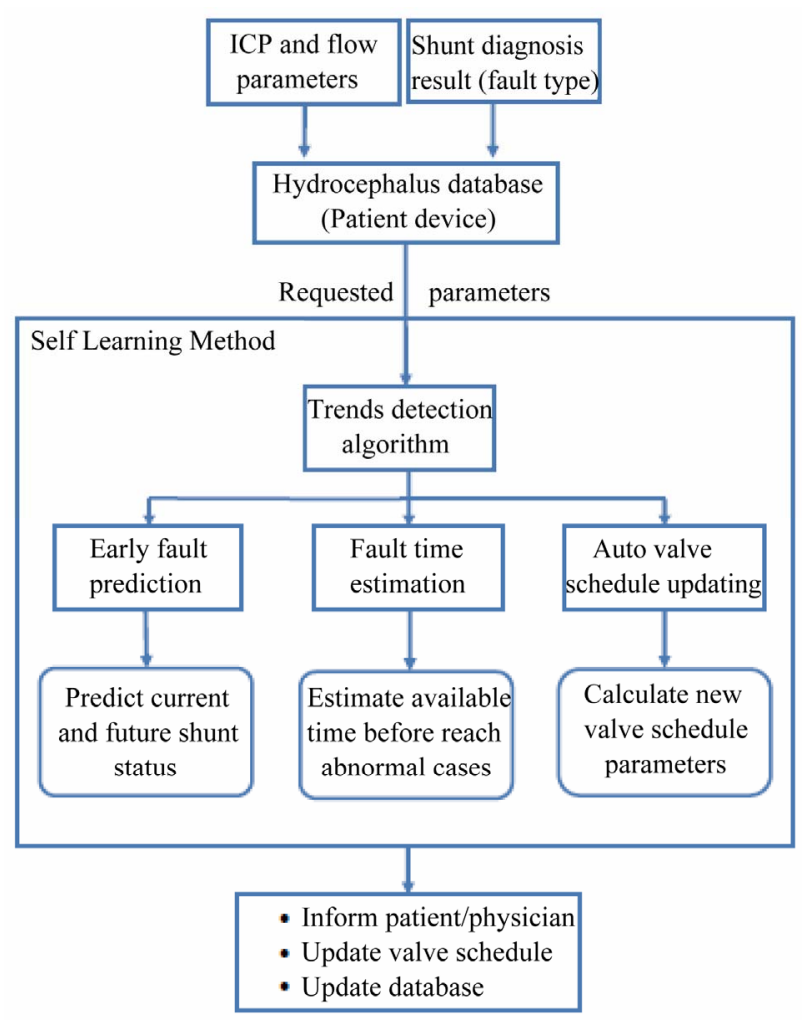

Figure 2. A block diagram of the proposed fault prediction and detection method. 
an autonomous modification of valve schedule when it is needed to deal with patients requirements, the second contribution of developing such method is to autonomously reflecting the effect of faults detection on valve schedule. An auto valve rescheduling approach is illustrated and simulated in this section. Such approach would deal with ICP rising by calculating and modifying the valve schedule. The modified schedule parameters (e.g. open duration, frequency of opening) are calculated based on the degree of changing in ICP due to shunt faults, increase/deacrease CSF production or increase/ deacrease in the natural drainage or blockage degree. Such approach would delay the risks accompanied with fault as long as possible.

\section{METHODOLOGY}

\subsection{Hydrocephalus Database}

A daily report, consisting samples of collected ICP readings, and their times of collection, is sent from implanted shunting system into external database which is hosted in the patient device. Based on valve schedule, a sample of ICP readings are collected to reflect the effect of open duration on ICP. The samples are taken for 6 -seconds window before opening and closing the valve.

The report consists of various parameters (e.g. mean ICP, fault type, mean absolute deviation (MAD) and valve flow). These parameters have strong correlations with expected shunt complications.

A self learning method utilizes this database for detecting any patterns and trends in ICP with time. Then a report would be generated that summarizes the results. A sample of such report is shown in Figure 3.

\subsection{Trends Detection and Fault Prediction}

The trend and pattern of mean ICP are detected every week. Three trends possibilities (positive, negative and no trend) are covered in this study. Based on the type of trend, a decision is made whether there is a possibility of shunt fault or improper valve schedule. A positive trend indicates a possibility of valve blockage or not enough

\begin{tabular}{|c|c|c|c|c|c|}
\hline Day & $\begin{array}{c}\text { Daily mean } \\
\text { ICP before } \\
(\mathrm{mmHg})\end{array}$ & $\mathrm{D}_{\text {off }}(\mathrm{min})$ & $\mathrm{D}_{\text {on }}(\min )$ & Period(min) & $\begin{array}{c}\text { Daily mean } \\
\text { ICP after } \\
(\mathrm{mmHg})\end{array}$ \\
\hline 1 & 11 & 20 & 10 & 30 & 6.18 \\
\hline 2 & 15.488 & 12 & 4 & 16 & 14.81 \\
\hline 3 & 17.643 & 18 & 6 & 24 & 15.86 \\
\hline 4 & 21 & 18 & 7 & 25 & 19.48 \\
\hline 5 & 21.99 & 18 & 7 & 24 & 19.85 \\
\hline 6 & 22.489 & 17 & 7 & 24 & 20.56 \\
\hline 7 & 24.545 & 15 & 6 & 22 & 22.2 \\
\hline
\end{tabular}

Figure 3. A sample of generated self learning report. valve opening. Albeit ICP is still within normal range, but there is systematic rise in ICP that would cross the normal range within certain period of time. Such trend detection helps encapsulating a fault in its early stages (preventive). Knowing that the fault at this stage cannot be detected by physician since its effect on the intracranial hydrodynamics is still minimal.

On the other hand, a negative trend indicates valve leakage or improper valve schedule for current patient condition. For both trends, there is a need to modify the valve schedule to delay the danger of propagation of such problem and gain time to solve the problem by physician.

An irregular is explained as instability in the production of CSF thus the patient might need more care.

\subsection{Fault Time Estimation}

Based on the detected trend, a daily rate of increase/ eacrease in the parameter value is calculated by taking the derivative of the trend with time.

For positive trend, the estimated time $\left(t_{A B}\right)$ for the effect of fault, e.g. valve blockage, to be observed through the abnormal intracranial hydrodynamics is calculated as follows

$$
\mathrm{t}_{\mathrm{AB}}=\frac{\overline{\mathrm{ICP}}_{\mathrm{UL}}-\overline{\mathrm{ICP}}_{\text {current }}}{\text { Rate of } \overline{\mathrm{ICP}} \text { change }}
$$

where $\mathrm{ICP}_{\mathrm{UL}}$ is the upper normal ICP limit, $\mathrm{ICP}_{\text {current }}$ is the mean ICP for current day.

$t_{\mathrm{AB}}$ indicates the time available before the ICP crosses the upper limit, thus if valve is revized within this time, patient's discomfort and subjection to danger can be avoided.

Furthermore, a full valve blockage limit can be estimated based on numerical simulation and time (tFL) for the valve to reach a full blockage limit can be calculated as follows,

$$
\mathrm{t}_{\mathrm{FL}}=\frac{\overline{\mathrm{ICP}}_{\mathrm{FL}}-\overline{\mathrm{ICP}}_{\text {current }}}{\text { Rate of } \overline{\mathrm{ICP}}_{\text {change }}}
$$

where $\mathrm{ICP}_{\mathrm{FL}}$ is the estimated mean ICP for full blockage case.

Then, the estimated time $\left(\mathrm{t}_{\mathrm{AL}}\right)$ for the effect of the detected fault, e.g. leakage, to be observed through the abnormal intracranial hydrodynamics can be calculated as follows,

$$
\mathrm{t}_{\mathrm{AL}}=\frac{\overline{\mathrm{ICP}}_{\text {current }}-\overline{\mathrm{ICP}}_{\mathrm{LL}}}{\text { Rate of } \overline{\mathrm{ICP}} \text { change }}
$$

where $\mathrm{ICP}_{\mathrm{LL}}$ is the lower normal ICP limit of mean ICP.

$\mathrm{t}_{\mathrm{AL}}$ gives an indication of the time available before ICP values crosses the lower limit, thus if valve is revized within this time, patient's discomfort and subjection to 
danger can be avoided.

Finally, estimated fault time can be utilized by the physician to plan for shunt revision thus reduce the risk of endangering patient's life. The outline of the proposed algorithm for early fault detection is illustrated in Figure 4.

\subsection{Auto Valve Schedule Updating}

The outcome of prediction and detection methods is used to autonomously modify the valve schedule to deal with the raised problem. A method is proposed that calculates the optimum valve schedule parameters, i.e. opening and closing durations of the valve. In this method, the system calculates the new valve schedule and updates it based on the effect of the fault on the intracranial hydrodynamics.

The valve schedule updating method is investigated to deal with the effect of both valve blockage and leakage faults. For valve blockage (the cause of positive trend) two cases were covered. In the first case, the detected trend is positive and the ICP is still within normal range. In this case, the time needed (dt) to reduce the rise in ICP to reach lower limit is calculated as shown in Figure 5.

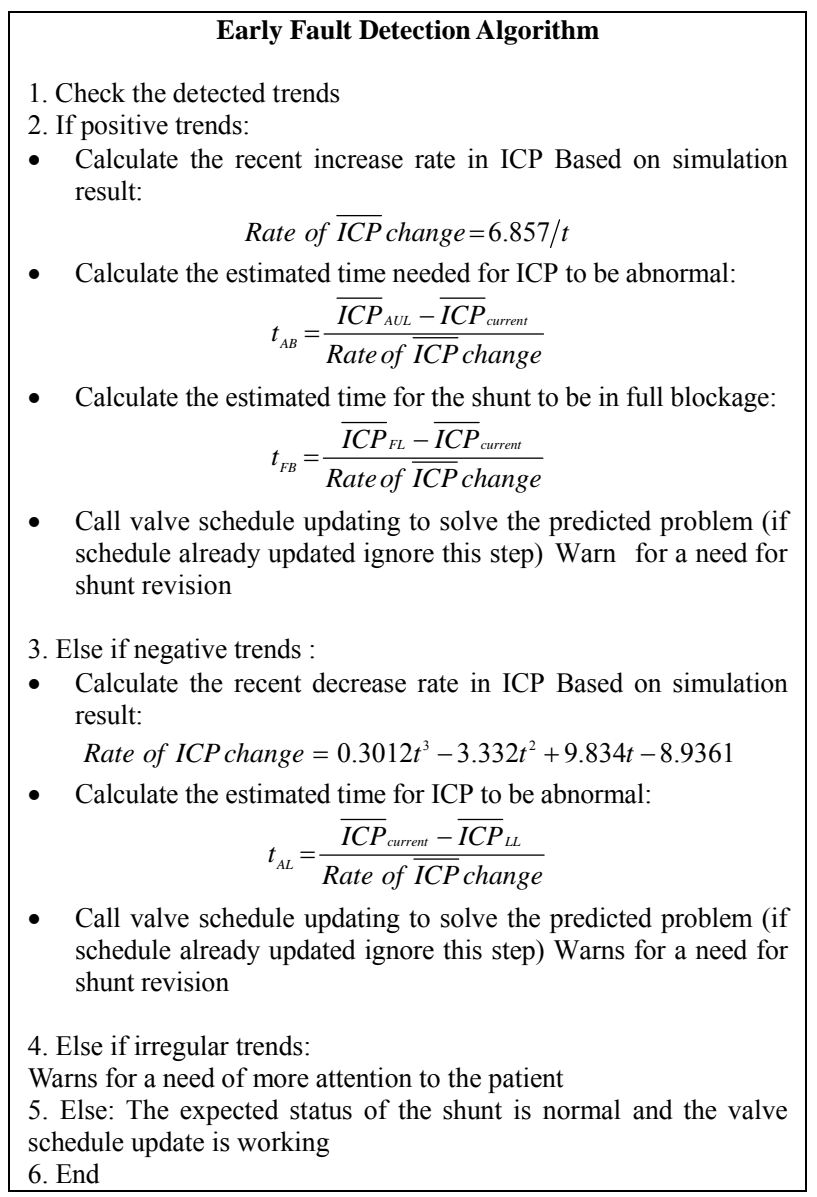

Figure 4. Early fault detection algorithm.
Valve Schedule Updating Algorithm

1. Positive trends with abnormal ICP case :

- Calculate the time needed time $\left(\mathrm{dt}_{\min }\right)$ to reduce the rise in ICP to reach upper limit:

$$
d t_{\min }=\frac{\text { Daily } \overline{I C P}_{\text {open }}-\text { Upper normal limit }}{\text { Rate of } \overline{I C P} \text { change }}
$$

- Calculate the time needed time $\left(\mathrm{dt}_{\max }\right)$ to reduce the rise in ICP to reach lower limit:

$$
d t_{\max }=\frac{\text { Daily } \overline{I C P}_{\text {open }}-\text { Lower normal lim it }}{\text { Rate of } \overline{I C P}_{\text {change }}}
$$

- Calculate the average time $\left(\mathrm{dt}_{\mathrm{avg}}\right)$ to reduce the rise in ICP to be within normal range:

$$
d t_{\text {avg }}=\frac{d t_{\max }+d t_{\min }}{2}
$$

- Based on the percentage of rising ICP comparing with the normal range, update valve schedule

1) If the rise in ICP is high:

New $d_{o}=d_{\max }$, where $d_{o}$ is valve open duration

2) else If the rise in ICP is low:

$$
\text { New } \mathrm{d}_{\mathrm{o}}=\mathrm{dt}_{\mathrm{avg}}
$$

- Follow the new valve schedule

2. Positive trend with normal ICP case:

- Calculate the time needed time (dt) to reduce the rise in ICP to reach lower limit:

$$
d t=\frac{\text { Daily } \overline{I C P}_{\text {open }}-\text { Lower normal limit }}{\text { Rate of } \overline{I C P} \text { change }}
$$

New $\mathrm{d}_{\mathrm{o}}<d t, \mathrm{~d}_{\mathrm{o}}$ is valve open duration

- Follow the new valve schedule

- End

Figure 5. The auto valve schedule updating algorithm (positive trend).

In the second case, the detected trend is positive and ICP is abnormal (above the upper limit). In this case, the proposed method involves several steps (illustrated in Figure 5). First, the time ( $\left.\mathrm{dt}_{\min }\right)$ needed to reduce the rise in ICP (due to blockage or fault) to the upper normal limit is calculated. Second, the time $\left(\mathrm{dt}_{\max }\right)$ needed to reduce the rise in ICP to the lower normal limit is also calculated. Then, the average $\left(\mathrm{dt}_{\text {average }}\right)$ is calculated, i.e. time needed for ICP to be within the normal range. These values are used for modifying valve schedule parameters in order to eliminate the effect of the gradual increase in the degree of the blockage as follows: If the rise in ICP is high (more than $20 \mathrm{mmHg}$ ), $\mathrm{dt}_{\max }$ is used as a new open duration of the valve. If the rise in ICP is low (less than $20 \mathrm{mmHg}), \mathrm{dt}_{\text {average }}$ is used as a new open duration of the valve.

For valve leakage, two cases also were covered. In the first case, the detected trend is negative and the ICP is still within normal range. In this case, the time needed (dt) to retrieve the drift in ICP to reach upper normal limit is calculated as shown in Figure 6. This value will be used as a new schedule parameter to regulate the valve based on the detected trend. 


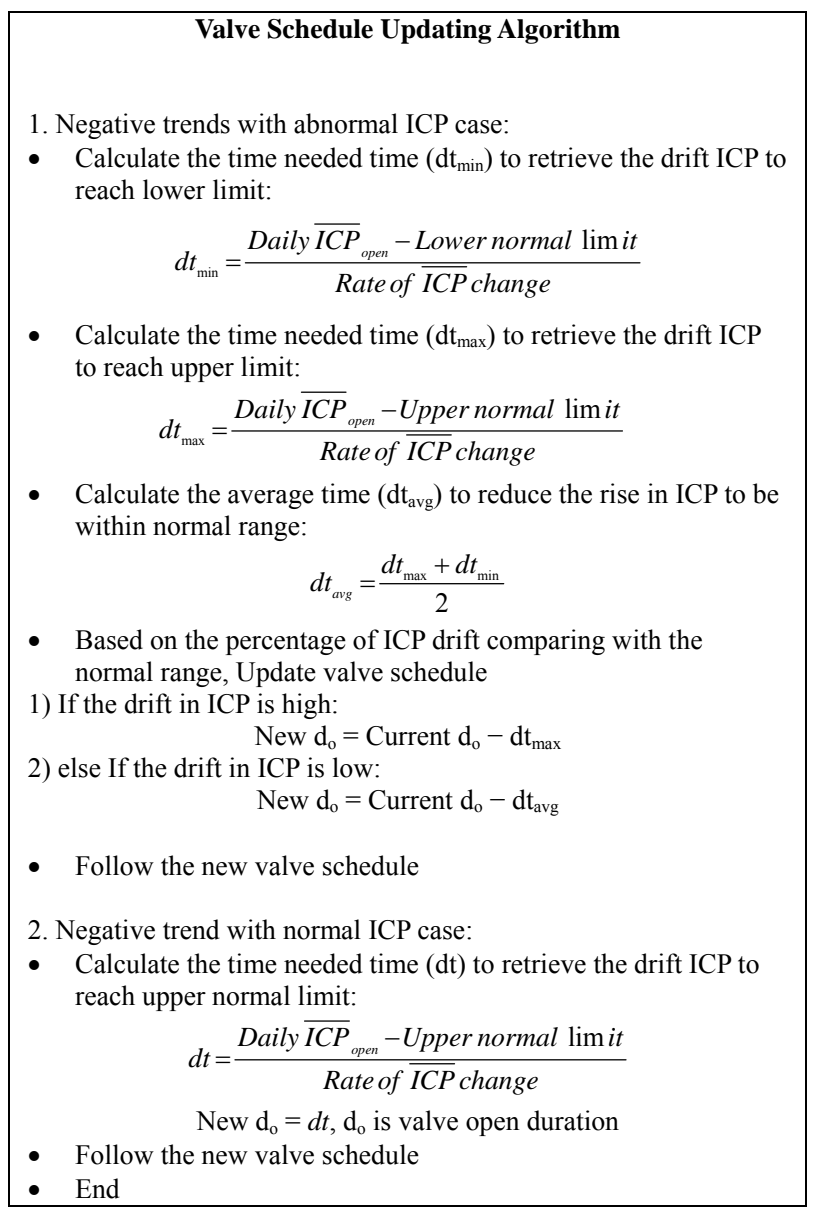

Figure 6. The auto valve schedule updating algorithm (negative trend).

In the second case, the detected trend is negative and the ICP is abnormal (below the lower limit). In this case, the proposed method involves the steps illustrated in Figure 6. First, the time needed $\left(\mathrm{dt}_{\min }\right)$ for ICP to reach lower limit is calculated. Second, the time needed $\left(\mathrm{dt}_{\max }\right)$ for ICP to reach upper limit is calculated. Then, the average time $\left(\mathrm{dt}_{\mathrm{avg}}\right)$ for ICP to be within normal range is also calculated. These values are used in modifying schedule parameters in order to eliminate the effect of the gradual decreases in ICP due to leakage, the modification is as follows: if the drift in ICP is high (greater than -5 $\mathrm{mmHg}),\left(\mathrm{d}_{\mathrm{tmax}}\right)$ is used as a new schedule parameter for the valve. If the drift in ICP is low (less than $-5 \mathrm{mmHg}$ ), $\mathrm{dt}_{\text {average }}$ is used as a new schedule parameter for the valve.

A performance measure was derived to evaluate the proposed method by calculating the total deviation of daily mean ICP from upper normal limit. This performance measure was calculated before and after applying the proposed technique for a specific time interval. The performance factor $(\mathrm{PF})$ is calculated as follows,

$$
\text { If }\left(\operatorname{ICP}_{\text {mean }}(\mathrm{d})-\mathrm{AUL}\right)>0 \text { then: }
$$

$$
\mathrm{P}_{\mathrm{AB1}}=\sum_{\mathrm{d}=1}^{\mathrm{n}}\left(\mathrm{ICP}_{\text {mean }}(\mathrm{d})-\mathrm{AUL}\right)
$$

Else

$$
\begin{aligned}
& \mathrm{P}_{\mathrm{AB} 2}=\sum_{\mathrm{d}=1}^{\mathrm{n}}\left(\operatorname{AUL}_{\left.-1 C P_{\text {mean }}(\mathrm{d})\right)}\right. \\
& \mathrm{P}_{\mathrm{AB}}=\mathrm{P}_{\mathrm{AB} 1}+\mathrm{P}_{\mathrm{AB} 2} \\
& \mathrm{PF}=1-\frac{\mathrm{P}_{\mathrm{AB} \text { (after) }}}{\mathrm{P}_{\mathrm{AB} \text { (before) }}}
\end{aligned}
$$

where, $\mathrm{P}_{\mathrm{AB}}$ is the summation of the difference between daily mean ICP and upper normal limit, AUL is the upper abnormal limit, $\mathrm{ICP}_{\text {mean }}$ is the average of ICP per day and $\mathrm{n}$ is the number of days. The closer to unity PF is, the better the performance of schedule modifying. PF values vary between 0 and 1 .

\subsection{Fault Modeling}

Numerical simulations was performed using Simulink ${ }^{\mathrm{TM}}$ model that reproduces intracranial hydrodynamics of hydrocephalus patients using historical ICP data [7]. This model was used as a dynamic environment to reflect the effect of adding a mechatronic valve on the intracranial hydrodynamics. A time-based valve schedule was used to control the opening and closing of this valve.

The most common shunt faults are valve blockage and leakage [8]. Thus, these faults were investigated in this study. The effect of such faults on the intracranial hydrodynamics were modelled using Simulink ${ }^{\mathrm{TM}}$ where they were incorporated into the intracranial hydrodynamics model. In addition, the model is modified to simulate different degrees of valve blockage and leakage. The shunting system was monitored for seven days where the ICP readings were collected and the ICP parameter values were calculated. The simulation started with fault-free shunt and then the faults were introduced.

Blockage degree is presented by a resistance $\left(R_{t}\right)$ that is made up of valve resistance and blockage resistance. The increase in $R_{t}$ due to blockage is assumed to have exponential relation with time that reflects the behaviour of blockage formation as follows,

$$
\mathrm{R}_{\mathrm{t}}=\mathrm{A} \times \mathrm{e}^{-\mathrm{Bt}}
$$

Two points were used to estimate the constants A and B of the model: 1) at zero time, it is assumed no fault, thus $R_{t}=$ valve resistance; 2 ) at the end of seven days it is assumed full blockage that $\mathrm{Rt}$ reaches seven times the valve resistance. As the result of substitution, $R_{t}$ can be model as follows,

$$
\mathrm{R}_{\mathrm{t}}=263.763 \times \mathrm{e}^{-1.93 \times 10^{-5} \mathrm{t}}
$$

After setting the value of $R_{t}$, the effect of the valve 
blockage on the performance of the shunt during seven days was noticed through the rise in ICP readings as well as reduction of valve flow.

The method for modifying the valve schedule was applied and the new opening valve durations was autonomously calculated for each blockage degree. These new open durations are applied in real time and the effect of such new schedule is illustrated in Figure 7. It can be noticed that, mean ICP stays for longer time within normal limit (less than $20 \mathrm{mmHg}$ ) compared with mean ICP before applying new schedule.

The proposed schedule modifying method was evaluated based on numerical simulation environment by comparing the effect of valve blockage on selected parameters before and after applying such method. The experimental work included four major steps: 1) generate a summary of important ICP and flow parameters; 2) use proposed methods for early fault detection and abnormality time estimation based on these parameters; 3 ) apply valve schedule updating method to find a shortterm solution to deal with the faults; and 4) finally, use numerical simulations to measure the performance of these methods.

\section{RESULTS AND DISCUSSION}

A novel approach is proposed to detect shunt faults in their early stages and work on delaying their effects till next shunt revision or till medical care is available. Numerical simulations were used to test proposed algorithm. Two types of faults, i.e. valve blockage and leakage, were simulated using Simulink ${ }^{\mathrm{TM}}$. Figures 8 and 9 illustrate ICP traces after incorporating the blockage and leakage faults, respectively. In addition, unstable increases/decrease of ICP was simulated as shown in Figure 10. Based on simulated ICP traces, trends of various ICP parameters were determined and used to detect the fault in its early stages and to monitor the effect of

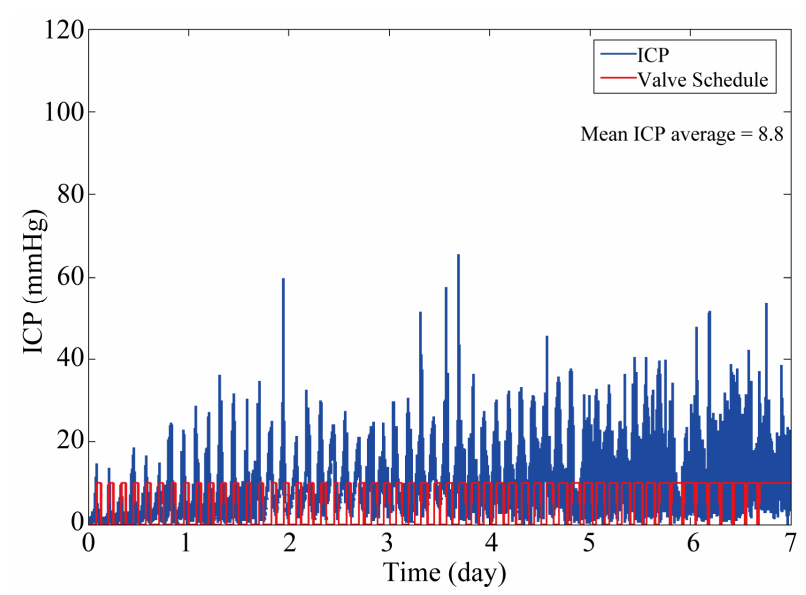

Figure 7. The effect of valve schedule updating method for selected days.

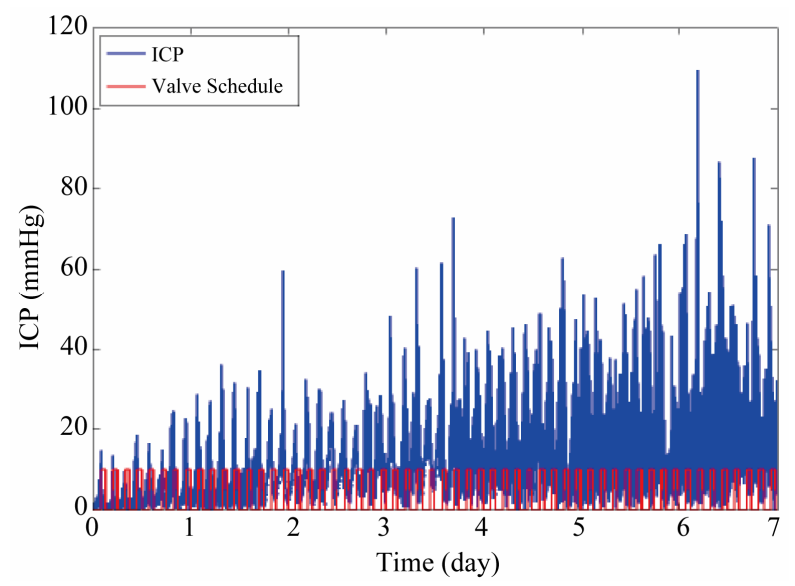

Figure 8. The effect of valve blockage on ICP trace.

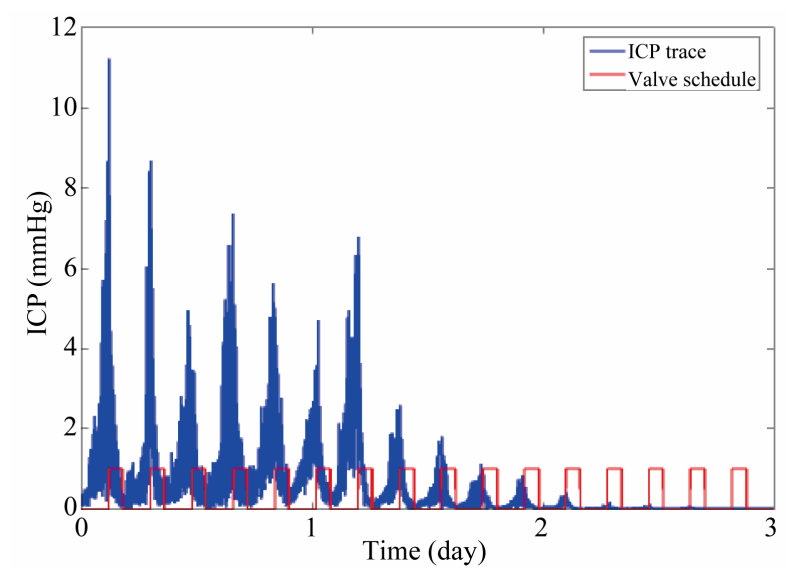

Figure 9. The effect of valve leakage on ICP trace.

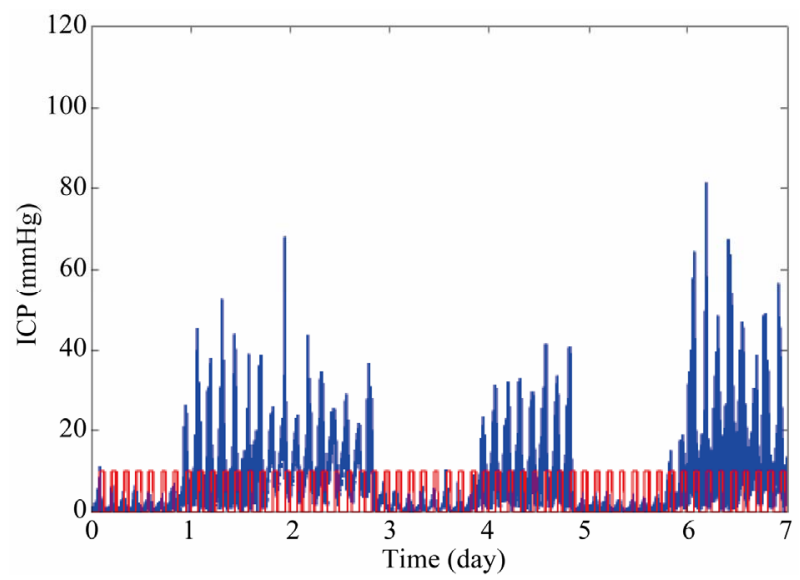

Figure 10. Unstable changing of ICP trace.

such fault on shunt performance step by step. Three types of trends were investigated; positive, negative, and no trends.

Figure 11 illustrates the predicted effect of valve blockage on the trends of daily average of mean ICP for seven days. This figure shows that there is a positive trend which means the ICP is rising albeit the valve sche- 


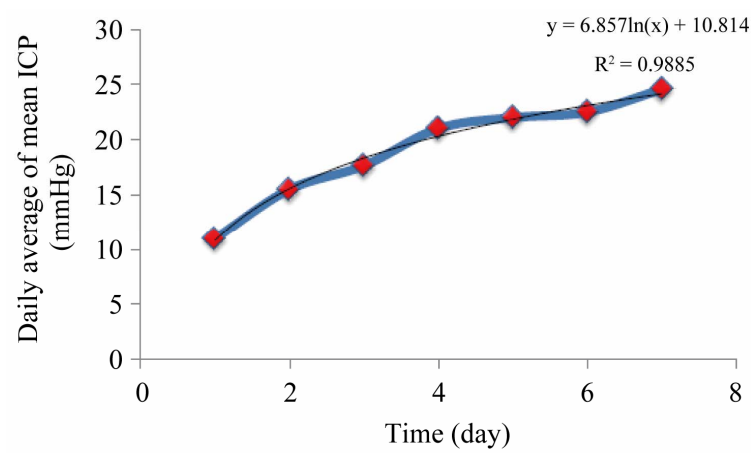

Figure 11. The trend of daily mean ICP due to blockage occurrence.

dule is used to regulate the valve. This increase is caused by the increase in the degree of valve blockage. Note that the trend was approximated to be logarithmic with $\mathrm{R}$-squared value equals 0.98 as follows,

$$
\overline{\mathrm{ICP}}=6.857 \times \ln (\mathrm{t})+10.841
$$

In case of positive trend, the daily rate of increase in mean ICP is calculated by deriving the model of the positive trend with respect to time and this gives the following equation,

$$
\text { Rate of ICP change }=\frac{6.857}{\mathrm{t}}
$$

where $t$ is the corresponding time (in days).

Figure 12 illustrates an example of negative trend. It shows the effect of valve leakage on the trends of daily average of mean ICP for seven days. It can be noticed that the daily mean ICP is continuously reduced due to valve fault.

This trend is approximated to be polynomial trendline as illustrated below. The value of mean ICP for this type of trend can be calculated based on time as follows,

$$
\begin{aligned}
\overline{\text { ICP }} & =0.0753 \mathrm{t}^{4}-1.107 \mathrm{t}^{3}+4.917 \mathrm{t}^{2} \\
& -8.9361 \mathrm{t}+6.7357
\end{aligned}
$$

The rate of drop in mean ICP $(\Delta \overline{\mathrm{ICP}} / \Delta \mathrm{t})$ due to valve leakage is calculated by taking the derivative of the negative trend as follows,

$$
\frac{\Delta \overline{\mathrm{ICP}}}{\Delta \mathrm{t}}=0.3012 \mathrm{t}^{3}-3.332 \mathrm{t}^{2}+9.8342 \mathrm{t}+8.9361
$$

Figure 13 illustrates an example of no trend. It can be seen that ICP changes in a series of irregular rise and drop cycles due to many reasons such as poor valve schedule.

In addition, the estimated times for ICP to reach the upper normal, lower normal and the full blockage limits are calculated. Table 1 summarizes these values for each corresponding day before and after applying the valve schedule updating algorithm.

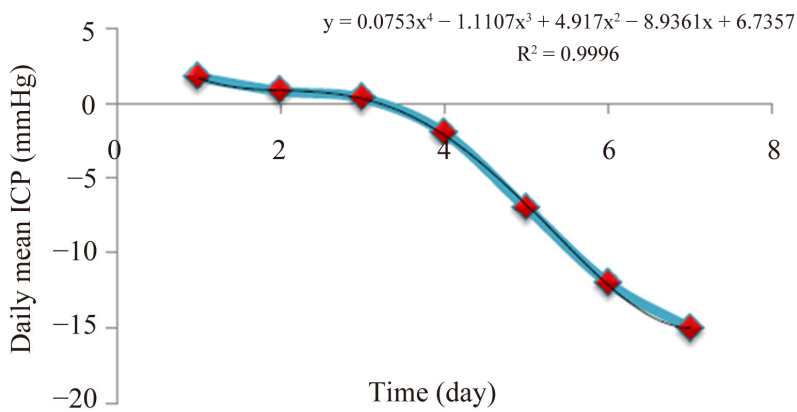

Figure 12. The change in the daily mean ICP due to leakage.

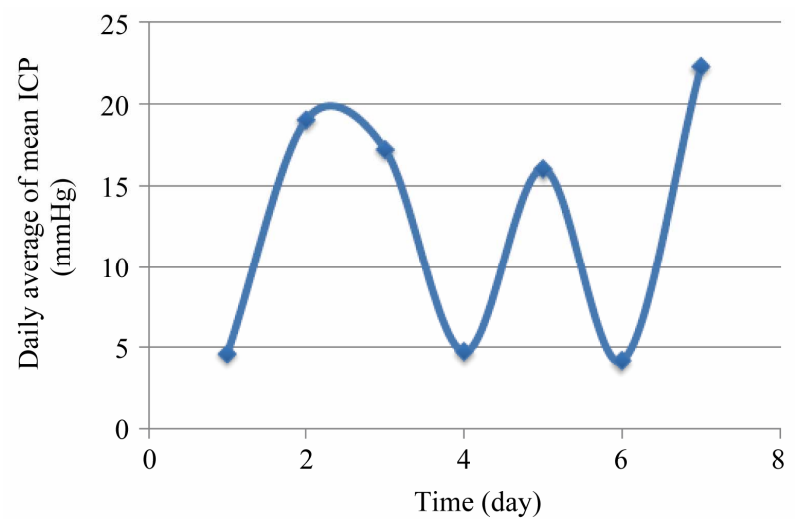

Figure 13. The irregular change in daily mean ICP.

A valve schedule updating method is used to temporarily deal with any early fault detection until the patient checked up by his/her physician. Such method would ensure that ICP is maintained within normal range for longer time compared with the time before applying such method.

In addition, valve schedule parameters were calculated based on the proposed valve schedule updating method and Table 2 summarizes these values for each corresponding day.

Based on mean ICP before and after applying the schedule updating method, it was found that the value of mean ICP was reduced due to applying the valve schedule updating method and it is maintained within normal range for longer time. It can be noticed that it succeeded in delaying the times needed to reach both abnormal and full blockage.

In addition, the performance factor (PF) in Eq.7 was used to evaluate the efficiency of this method. PF value was 0.94 .

\section{CONCLUSIONS}

A novel approach is proposed for detecting valve faults at early stages, estimating the time required for the ICP to be abnormal and estimating the time required for the valve to reach full blockage case. Thus plan revision schedules for the valve. 
Table 1. The estimated times for the ICP to reach the upper normal and the full blockage limits: (a) before applying valve schedule updating algorithm; (b) after applying the proposed algorithm.

(a)

\begin{tabular}{ccccc}
\hline Day & Daily mean ICP (mmHg) & $\begin{array}{c}\text { Rate of daily change in mean ICP } \\
(\mathbf{m m H g} / \mathbf{d a y})\end{array}$ & $\begin{array}{c}\text { Time (day) to reach } \\
\text { AUL }\left(\mathbf{t}_{\mathbf{A B}}\right)\end{array}$ & $\begin{array}{c}\text { Time (day) to reach FUL } \\
\left(\mathbf{t}_{\mathrm{FL}}\right)\end{array}$ \\
\hline 1 & 11.00 & 6.86 & 0.72 & 2.77 \\
2 & 15.40 & 3.43 & 0.14 & 4.23 \\
3 & 17.64 & 2.29 & -0.71 & 5.40 \\
4 & 21.00 & 1.71 & -2.91 & 5.25 \\
5 & 21.99 & 1.37 & -4.36 & 5.84 \\
6 & 22.48 & 1.14 & -5.67 & 6.57 \\
7 & 24.54 & 0.98 & -8.72 & 5.56 \\
\hline
\end{tabular}

(b)

\begin{tabular}{ccccc}
\hline Day & Daily mean ICP (mmHg) & $\begin{array}{c}\text { Rate of daily change in mean ICP } \\
(\mathbf{m m H g} / \mathbf{d a y})\end{array}$ & $\begin{array}{c}\text { Time (day) to reach } \\
\text { AUL }\left(\mathbf{t}_{\mathrm{AB}}\right)\end{array}$ & $\begin{array}{c}\text { Time (day) to reach FUL } \\
\left(\mathbf{t}_{\mathrm{FL}}\right)\end{array}$ \\
\hline 1 & 11.00 & 6.85 & 0.72 & 2.77 \\
2 & 15.48 & 3.42 & 0.14 & 4.23 \\
3 & 17.64 & 2.28 & -0.71 & 5.40 \\
4 & 18.00 & 1.71 & -1.16 & 7.00 \\
5 & 17.50 & 1.37 & -1.09 & 9.11 \\
6 & 16.99 & 1.14 & -0.86 & 11.38 \\
7 & 16.81 & 0.97 & -0.82 & 13.46 \\
\hline
\end{tabular}

Table 2. The valve schedule parameters based on proposed valve schedule updating method.

\begin{tabular}{cccccc}
\hline Day & $\begin{array}{c}\text { Daily mean ICP before } \\
(\mathbf{m m H g})\end{array}$ & $\mathbf{D}_{\text {off }}(\mathbf{m i n})$ & $\mathbf{D}_{\text {on }}(\mathbf{m i n})$ & Period (min) & $\begin{array}{c}\text { Daily mean ICP after } \\
(\mathbf{m m H g})\end{array}$ \\
\hline 1 & 11.00 & 20 & 10 & 30 & 11.00 \\
2 & 15.49 & 20 & 10 & 30 & 15.49 \\
3 & 17.64 & 20 & 10 & 30 & 17.64 \\
4 & 21.00 & 17 & 13 & 30 & 18.00 \\
5 & 21.99 & 14 & 16 & 30 & 17.50 \\
7 & 22.49 & 11 & 19 & 30 & 30 \\
\hline
\end{tabular}

In addition, the method proposed would autonomously manage the valve schedule, thus temporarily delay the effect of the detected fault and reduce the risks of such faults on patient's life and reduce his/her suffering. In addition, the patient's suffering due to valve revisions would be reduced by applying such method.

Furthermore, hydrocephalus treatment information as well as shunt diagnosis information can be used to build a useful hydrocephalus database for better understanding of hydrocephalus and shunt malfunctions. That can lead to improve shunt performance and at the same time optimize the treatment.

\section{REFERENCES}

[1] Association for Spina Bifida Hydrocephalus (2009) Hydrocephalus. http://www.asbah.org/.

[2] Albright, A., Haines, S. and Taylor, F. (1988) Function of parietal and frontal shunts in childhood hydrocephalus. Journal of Neurosurgery, 69, 883- 886. doi:10.3171/jns.1988.69.6.0883

[3] Piatt, J. (1995) Cerebrospinal fluid shunt failure: Late is 
different from early. Pediatric Neurosurgery, 23, 133-139. doi:10.1159/000120950

[4] Piatt, J. and Carlson, C. (1993) A search for determinants of cerebrospinal fluid shunt survival: Retrospective analysis of a 14-year institutional experience. Pediatric Neurosurgery, 19, 233-241. doi:10.1159/000120738

[5] Villavicencio, A., Leveque, J., McGirt, M., Hopkins, J., Fuchs, H. and George, T. (2003) Comparison of revision rates following endoscopically versus nonendoscopically placed ventricular shunt catheters. Surgical Neurology, 59, 375-379. doi:10.1016/S0090-3019(03)00070-3

[6] Koyrakh, L. (2008) Data compression for implantable medical devices. Computers in Cardiology, 35.
[7] Momani, L. (2010) Intelligent system for personalised management and treatment of hydrocephalus. Ph.D. Thesis, University of Liverpool, Liverpool.

[8] Momani, L., Alkharabsheh, A., Al-Zuibi, N. and Al-Nuaimy, W. (2009) Instantiating a mechatronic valve schedule for a hydrocephalus shunt. Conference Proceedings: IEEE Engineering in Medicine and Biology Society, Minneapolis, 3-6 September, 2009, 749-752.

[9] Aoki, N. (1990) Lumboperitoneal shunt: Clinical applications, complications and comparison with venticuloperitoneal shunt. Neurosurgery, 26, 998-1004. doi:10.1227/00006123-199006000-00013 